\title{
Optimization approach for the computation of magnetohydrostatic coronal equilibria in spherical geometry
}

\author{
T. Wiegelmann ${ }^{1}$, T. Neukirch ${ }^{2}$, P. Ruan ${ }^{1}$, and B. Inhester ${ }^{1}$ \\ 1 Max-Planck-Institut für Sonnensystemforschung, Max-Planck-Strasse 2, 37191 Katlenburg-Lindau, Germany \\ e-mail: wiegelmann@mps.mpg.de \\ 2 School of Mathematics and Statistics, University of St. Andrews, St. Andrews, KY16 9SS, UK
}

Received 9 July 2007 / Accepted 3 September 2007

ABSTRACT

\begin{abstract}
Context. This paper presents a method which can be used to calculate models of the global solar corona from observational data. Aims. We present an optimization method for computing nonlinear magnetohydrostatic equilibria in spherical geometry with the aim to obtain self-consistent solutions for the coronal magnetic field, the coronal plasma density and plasma pressure using observational data as input.

Methods. Our code for the self-consistent computation of the coronal magnetic fields and the coronal plasma solves the non-forcefree magnetohydrostatic equilibria using an optimization method. Previous versions of the code have been used to compute nonlinear force-free coronal magnetic fields from photospheric measurements in Cartesian and spherical geometry, and magnetostaticequilibria in Cartesian geometry. We test our code with the help of a known analytic 3D equilibrium solution of the magnetohydrostatic equations. The detailed comparison between the numerical calculations and the exact equilibrium solutions is made by using magnetic field line plots, plots of density and pressure and some of the usual quantitative numerical comparison measures.

Results. We find that the method reconstructs the equilibrium accurately, with residual forces of the order of the discretisation error of the analytic solution. The correlation with the reference solution is better than $99.9 \%$ and the magnetic energy is computed accurately with an error of $<0.1 \%$.

Conclusions. We applied the method so far to an analytic test case. We are planning to use this method with real observational data as input as soon as possible.
\end{abstract}

Key words. Sun: corona - Sun: magnetic fields - methods: numerical

\section{Introduction}

In the recent past, numerical methods based on optimization principles have been used for a number of problems associated with the calculation of solar MHD equilibria. Wheatland et al. (2000) were the first to suggest the use of an optimization method to calculate nonlinear force-free fields in the corona from photospheric measurements. Since then the optimization method has been extended in various ways, for example by improving certain aspects of the original method for force-free fields (e.g. Wiegelmann 2004), by introducing additional features such as plasma pressure into the method (e.g. Wiegelmann $\&$ Neukirch 2006) or by reformulating the method in other geometries (e.g. Wiegelmann 2007).

Optimization methods have the advantage of being conceptually straightforward and are reasonably easy to implement (Wheatland et al. 2000; Wiegelmann \& Neukirch 2003; Inhester \& Wiegelmann 2006, see e.g.). At the moment they also seem to be very competitive in terms of computational efficiency (e.g Schrijver et al. 2006; Metcalf et al. 2007). A slight disadvantage compared to, for example, the Grad-Rubin method (e.g. Amari et al. 1997; Wheatland 2006; Inhester \& Wiegelmann 2006; Amari et al. 2006) is that they still lack the same degree of rigorous mathematical basis existing for other methods.

In the present paper we describe a further extension of the optimization method to calculate magnetohydrostatic (MHS) equilibria (including pressure and gravity) in spherical geometry. This is important for calculating global models of the corona including information going beyond just the structure of the magnetic field. In Sect. 2 we describe the basic equations of the optimization method for problem in hand. We then give a brief description of the analytical 3D MHS equilibria that we use to test the numerical code in Sect. 3 and present the test results in Sect. 4. Our conclusions are presented in Sect. 5.

\section{Basic equations}

The magnetohydrostatic (MHS) equations are given by

$(\nabla \times \boldsymbol{B}) \times \boldsymbol{B}-\mu_{0} \nabla p-\mu_{0} \rho \nabla \Psi=0$

$$
\nabla \cdot \boldsymbol{B}=0 \text {, }
$$

where $\boldsymbol{B}$ is the magnetic field, $p$ the plasma pressure, $\rho$ the mass density and $\Psi=-\frac{G M_{\mathrm{s}}}{r}$ the gravitational potential with the gravitational constant $G$, the solar mass $M_{\mathrm{s}}$ and the distance from the sun's center $r$. We do not assume an equation of state for the coronal plasma, but leave $p$ and $\rho$ to be independent quantities. To find a magnetic field $\boldsymbol{B}$, plasma pressure $p$ and plasma density $\rho$ satisfying Eqs. (1) and (2), we follow the spirit of the previous optimization methods (e.g. Wheatland et al. 2000; 
Wiegelmann \& Inhester 2003; Wiegelmann 2004; Wiegelmann $\&$ Neukirch 2006) and define the functional

$$
\begin{aligned}
L(\boldsymbol{B}, p, \rho)= & \int\left[\frac{\left|(\nabla \times \boldsymbol{B}) \times \boldsymbol{B}-\mu_{0} \nabla p-\mu_{0} \rho \nabla \Psi\right|^{2}}{B^{2}}\right. \\
& \left.+|\nabla \cdot \boldsymbol{B}|^{2}\right] r^{2} \sin \theta \mathrm{d} r \mathrm{~d} \theta \mathrm{d} \phi .
\end{aligned}
$$

It is obvious that Eqs. (1) and (2) are satisfied if $L=0$. Here $\boldsymbol{B}$ is a vector field, but not necessarily a solenoidal magnetic field during the iteration. The numerical method is based on an iterative scheme to minimize the functional $L$. To simplify the mathematical derivation we define the quantities

$\boldsymbol{\Omega}_{a}=B^{-2}\left[(\nabla \times \boldsymbol{B}) \times \boldsymbol{B}-\mu_{0} \nabla p-\mu_{0} \rho \nabla \Psi\right]$

$\boldsymbol{\Omega}_{b}=B^{-2}[(\nabla \cdot \boldsymbol{B}) \boldsymbol{B}]$,

and rewrite $L$ as

$L=\int_{V}\left[B^{2} \Omega_{a}^{2}+B^{2} \Omega_{b}^{2} r^{2}\right] \sin \theta \mathrm{d} r \mathrm{~d} \theta \mathrm{d} \phi$.

Taking the derivative of $L$ with respect to an iteration parameter $t$, where $\boldsymbol{B}, p, \rho$ are assumed to depend upon $t$, we obtain

$$
\begin{aligned}
\frac{1}{2} \frac{\mathrm{d} L}{\mathrm{~d} t}= & -\int_{V} \frac{\partial \boldsymbol{B}}{\partial t} \cdot \boldsymbol{F} \mathrm{d} V+\int_{V} \frac{\partial p}{\partial t} \mu_{0} \nabla \cdot \boldsymbol{\Omega}_{a} \mathrm{~d} V \\
& -\int_{V} \frac{\partial \rho}{\partial t} \mu_{0} \boldsymbol{\Omega}_{a} \cdot \nabla \Psi \mathrm{d} V-\int_{S} \frac{\partial \boldsymbol{B}}{\partial t} \cdot \boldsymbol{G} \mathrm{d} S \\
& -\int_{S} \frac{\partial p}{\partial t} \mu_{0} \boldsymbol{\Omega}_{a} \cdot \mathrm{d} \boldsymbol{S},
\end{aligned}
$$

where

$$
\begin{aligned}
\boldsymbol{F}= & \nabla \times\left(\boldsymbol{\Omega}_{a} \times \boldsymbol{B}\right)-\boldsymbol{\Omega}_{a} \times(\nabla \times \boldsymbol{B}) \\
& +\nabla\left(\boldsymbol{\Omega}_{b} \cdot \boldsymbol{B}\right)-\boldsymbol{\Omega}_{b}(\nabla \cdot \boldsymbol{B})+\left(\Omega_{a}^{2}+\Omega_{b}^{2}\right) \boldsymbol{B}
\end{aligned}
$$

and

$\boldsymbol{G}=\hat{\boldsymbol{n}} \times\left(\boldsymbol{\Omega}_{a} \times \boldsymbol{B}\right)-\hat{\boldsymbol{n}}\left(\boldsymbol{\Omega}_{b} \cdot \boldsymbol{B}\right)$.

Assuming that

$$
\begin{aligned}
& \frac{\partial \boldsymbol{B}}{\partial t}=\mu \boldsymbol{F} \\
& \frac{\partial p}{\partial t}=-v \mu_{0} \nabla \cdot \boldsymbol{\Omega}_{a} \\
& \frac{\partial \rho}{\partial t}=\xi \mu_{0} \boldsymbol{\Omega}_{a} \cdot \nabla \Psi=\xi \mu_{0} \frac{G M_{\mathrm{s}}}{r^{2}} \boldsymbol{\Omega}_{a} \cdot \boldsymbol{e}_{r}
\end{aligned}
$$

with positive definite parameters $\mu, v$ and $\xi$ and that the boundary integrals vanish, one can easily see that $L$ is monotonically decreasing with $t$ (note that this does not necessarily imply that $L$ tends to zero).

Discretized versions of Eqs. (10) to (12), together with appropriate boundary conditions, form the basis for the numerical scheme. The boundary conditions have to be consistent with the assumption that the boundary integrals vanish, for example, by keeping the magnetic field, pressure and density fixed on the boundaries during the iteration. For testing the method in this paper we shall take these boundary conditions from the known exact solution. For practical applications these would have to come from observational data. We remark that due to the introduction of additional forces the constraints on the consistency of the boundary conditions for the magnetic field are somewhat different from the force-free case. However, the general theory of magnetohydrostatic equilibria requires for example that the pressure has the same value at both foot points of a closed field line under the general conditions assumed in the present paper. This is similar to the Cartesian case discussed by Wiegelmann \& Neukirch (2006), where the pressure equation was forward integrated along field lines using an upwind method from one foot point to the other (in the test case described later we make use of the property that the pressure is known to be consistent).

In the numerical implementation based on this method one has to choose the product of the time-step $\Delta t$ with the three numerical parameters $\mu, v$ and $\xi$. Usually these products have to be small enough to achieve convergence and this is ensured by an adaptive time-step control. In this paper we have chosen the three parameters (multiplied by $\Delta t$ ) to have the same values on all grid points. Previous experience with applying a similar method to force-free magnetic fields in spherical geometry (Wiegelmann 2007) showed that choosing the same values for the entire box can lead to long computing times in the polar regions due to the distortion of the numerical grid in spherical polar coordinates towards the poles (note that the poles $\theta=0$ and $\theta=\pi$ are excluded from the computational domain). This could in principle be compensated by allowing for a spatial variation of the iteration parameters.

\section{3D MHS equilibria}

We use the exact 3D MHS equilibrium in spherical coordinates presented by Neukirch (1995) to test our code (called case II in his paper). In his paper, Neukirch (1995) extended earlier work by Bogdan \& Low (1986) on exact 3D MHS equilibria in spherical coordinates. The general method was first found by Low (1985) (previous closely related work can also be found in Low 1982; and Low 1985) for Cartesian coordinates and developed further in Low (1991, 1992, 1993a,b, 2005). The method relies on the presence of an external force (in our case gravitation) and the basic assumption that the electric currents flow only in surface direction perpendicular to the direction of the external force. The additional assumption that the dependence of the current density on the spatial coordinates has a special form involving the magnetic field component along the direction of the external force (in our case that is the radial component of $\boldsymbol{B})$ leads to a linear equation for that magnetic field component. The plasma density and pressure are determined from the force balance equation.

Neukirch (1995) has extended this method by including a current density component of the constant- $\alpha$ type ( $\alpha \boldsymbol{B}$ with $\alpha$ constant), which also allows components of the current density in the direction of the external force. It is important to emphasize that this does not mean that the total field-aligned current density is of the linear force-free type, because the other component of the current density also has a field-aligned part.

The formulation by Neukirch (1995) basically reduces the mathematical problem to the solution of an equation similar to a Schrödinger equation. A slightly simpler formulation of the same problem was given by Neukirch \& Rastätter (1999) and some analytical solutions (in Cartesian coordinates) with a nonlinear relationship between the current density and the magnetic field were found by Neukirch (1997). A formulation using Green's functions was given by Petrie \& Neukirch (2000).

Since the magnetic field given in Eqs. (45)-(47) of Neukirch (1995) contain a number of typographical errors, we repeat the 
correct field components here. As in Neukirch (1995) we define the functions

$$
\begin{aligned}
& f_{1}(r)=\frac{\cos q+q \sin q}{\cos q_{0}+q_{0} \sin q_{0}} \\
& f_{2}(r)=\frac{\left(3-q^{2}\right) \cos q+3 q \sin q}{\left(3-q_{0}^{2}\right) \cos q_{0}+3 q_{0} \sin q_{0}}
\end{aligned}
$$

with

$q=\alpha(r+a), \quad q_{0}=\alpha\left(r_{0}+a\right)$.

We then obtain ${ }^{1}$

$$
\begin{aligned}
B_{r}= & A_{10} \frac{r_{0}^{2}}{r^{2}} \frac{r_{0}+a}{r+a} f_{1}(r) Y_{1}^{0} \\
& -\frac{1}{2} A_{21} \frac{r_{0}^{2}}{r^{2}} \frac{\left(r_{0}+a\right)^{2}}{(r+a)^{2}} f_{2}(r)\left(Y_{2}^{1}-Y_{2}^{-1}\right) \\
B_{\theta}= & \frac{1}{2} A_{10} \frac{r_{0}^{2}}{r} \frac{r_{0}+a}{r+a}\left(\frac{\mathrm{d} f_{1}}{\mathrm{~d} r}-\frac{f_{1}}{r+a}\right) \frac{\partial}{\partial \theta} Y_{1}^{0} \\
& -\frac{1}{12} A_{21} \frac{r_{0}^{2}}{r} \frac{\left(r_{0}+a\right)^{2}}{(r+a)^{2}}\left(\frac{\mathrm{d} f_{2}}{\mathrm{~d} r}-\frac{2 f_{2}}{r+a}\right) \frac{\partial}{\partial \theta}\left(Y_{2}^{1}-Y_{2}^{-1}\right) \\
& -\frac{1}{12} A_{21} \alpha \frac{r_{0}^{2}}{r} \frac{\left(r_{0}+a\right)^{2}}{(r+a)^{2}} f_{2}(r) \frac{1}{\sin \theta} \frac{\partial}{\partial \phi}\left(Y_{2}^{1}-Y_{2}^{-1}\right) \\
B_{\phi}= & -\frac{1}{2} A_{10} \alpha \frac{r_{0}^{2}}{r} \frac{r_{0}+a}{r+a} f_{1}(r) \frac{\partial}{\partial \theta} Y_{1}^{0} \\
& +\frac{1}{12} A_{21} \frac{r_{0}^{2}}{r} \frac{\left(r_{0}+a\right)^{2}}{(r+a)^{2}} f_{2}(r) \frac{\partial}{\partial \theta}\left(Y_{2}^{1}-Y_{2}^{-1}\right) \\
- & \frac{1}{12} A_{21} \frac{r_{0}^{2}}{r} \frac{\left(r_{0}+a\right)^{2}}{(r+a)^{2}}\left(\frac{\mathrm{d} f_{2}}{\mathrm{~d} r}-\frac{2 f_{2}}{r+a}\right) \frac{1}{\sin \theta} \frac{\partial}{\partial \phi}\left(Y_{2}^{1}-Y_{2}^{-1}\right) .
\end{aligned}
$$

These expression tend to the solution presented as case III in Bogdan \& Low (1986) in the limit $\alpha=0$. The magnetic field is shown in the top panel of Fig. 1. The solution is completed by expressions for the pressure and the density as given by Bogdan \& Low (1986) and Neukirch (1995).

Neukirch (1995) followed Bogdan \& Low (1986) and normalized the radial coordinate $r$ at a radius of $1.5 R_{\odot}$. In the present paper, we deviate from this and normalize the radial coordinate at a radius of $1 R_{\odot}$, because we want to impose our boundary conditions at the solar surface $\left(=1 R_{\odot}\right)$. We then carry out our numerical calculation on a box which has an inner boundary at $1 R_{\odot}$ and an outer boundary at $(1.0+\pi / 2) R_{\odot} \approx$ $2.57 R_{\odot}$. We use a grid with 20 points in the radial direction, 40 points in the latitudinal direction and 80 points in the longitudinal direction. We exclude the polar regions for numerical reasons (see discussion in Sect. 2) and extend the numerical box in $\theta$ only from $11.25^{\circ}$ to $168.75^{\circ}$.

As parameters we choose the parameter $a$, which determines the influence of the non-magnetic forces on the equilibrium, to have the value $a=0.2$, and we choose the force-free parameter $\alpha$ to have the value $\alpha=0.5$. Internally our code normalizes the length scale with one solar radius, the magnetic field strength with the average radial magnetic field strength on the photosphere $B_{\text {ave }}$, the pressure with $\tilde{p}=\mu_{0} p / B_{\text {ave }}^{2}$ and the mass density with $\tilde{\rho}=\mu_{0} G M_{\mathrm{s}} \rho / R_{\mathrm{S}} B_{\text {ave }}^{2}$.

${ }^{1}$ Compared to Neukirch (1995) these expression have been corrected in the following way: a) a factor $1 / 2$ has been included in the second term of $\left.B_{r}, \mathrm{~b}\right)$ a factor $1 / \sin \theta$ has been removed from the second term of $B_{\phi}$ and c) the sign of the third term of $B_{\phi}$ is negative.
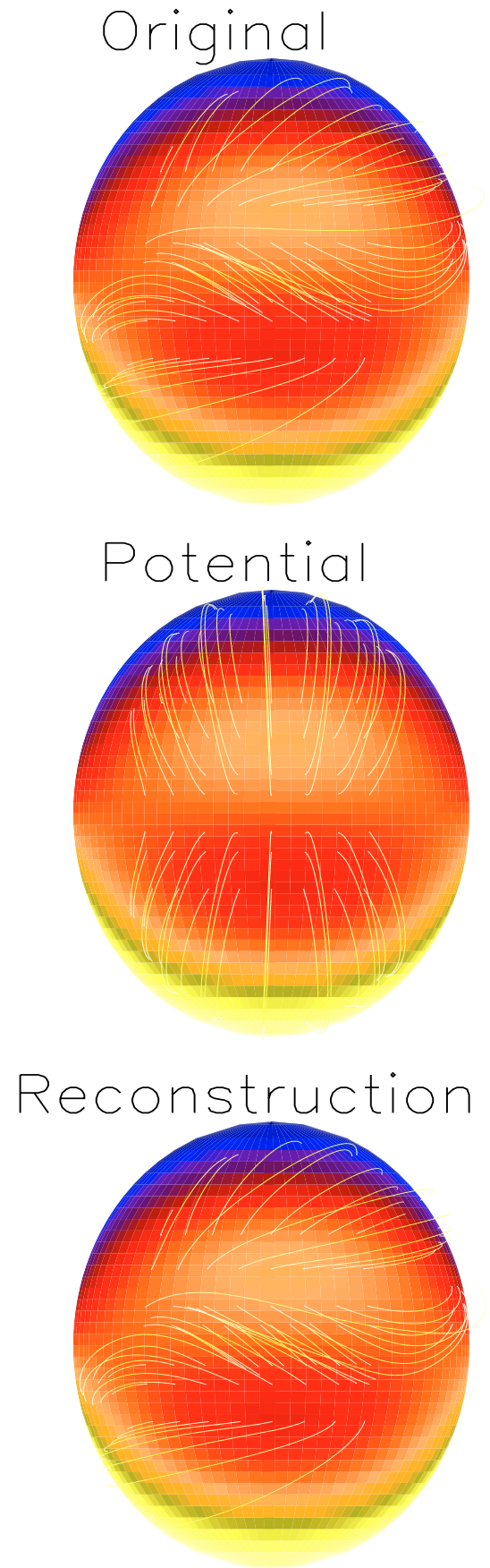

Fig. 1. Magnetic field lines for the exact analytical solution, a potential field with the same $B_{r}$ on the photospheric boundary $\left(r=R_{\odot}\right)$ and the magnetic field obtained by the optimization method. The color coding corresponds to the value of $B_{r}$ on the photosphere (yellow: positive, blue: negative) and the disk center corresponds to $\phi=180^{\circ}$. The potential field is used as the initial field for the numerical calculation and clearly has a different connectivity from the exact analytical solution. The reconstructed solution matches the analytical solution down to plotting precision, except for one equatorial field line.

\section{Results}

The numerical code based on the optimization method described in Sect. 2 has been run with initial conditions given by a potential field calculated from just the $B_{r}$ component of the above exact solution on the boundaries. The initial conditions for density and pressure are as in a stratified atmosphere. This configuration 


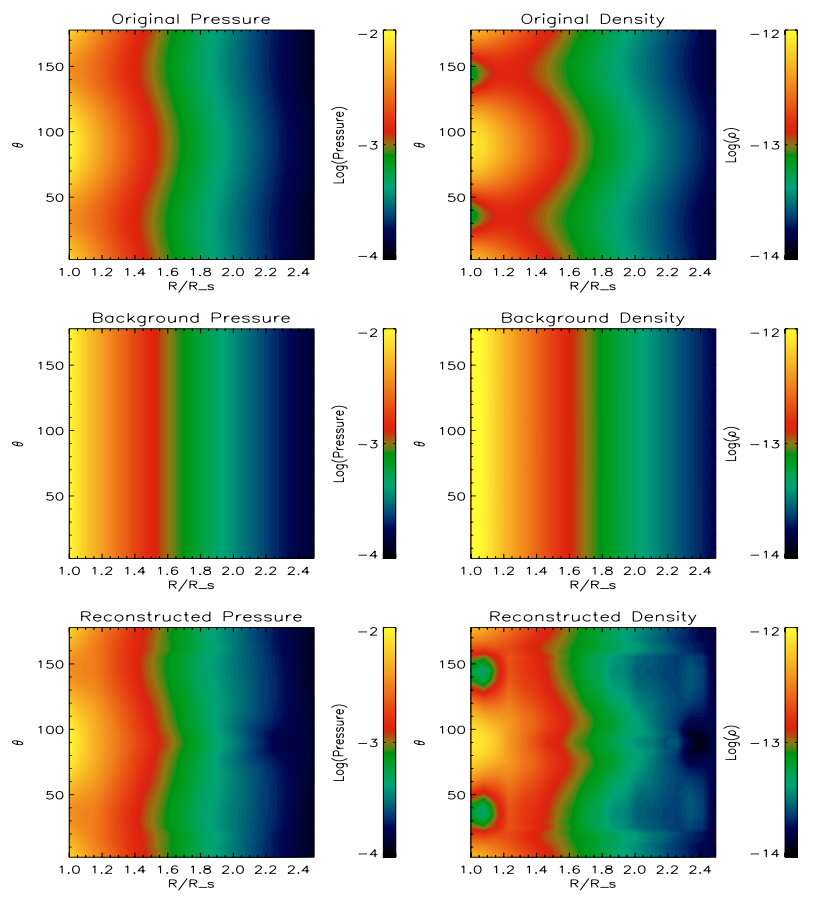

Fig. 2. Plots of the spatial variation of the pressure $p$ (left column) and density $\rho$ (right column) in logarithmic scaling in the $r-\theta$-plane for $\phi=0$. The top, center and bottom panels correspond to the exact solution, the spherically symmetric stratified atmosphere used as initial state for the iteration (also identical to the background atmosphere used in the analytical solution) and the result of the numerical calculation. The main features of the analytical solutions are clearly recovered by the numerical method, but some small differences are still present.

balances the radial pressure gradient and the gravity force. Consequently the initial plasma is structured only in the radial direction and $p$ and $\rho$ are invariant in $\theta$ and $\phi$. We use the exact solution to prescribe the boundary conditions for $\boldsymbol{B}, p, \rho$. Our code solves the magnetohydrostatic Eqs. (1), (2) in the computational box with respect to these boundary conditions. To evaluate the performance of our code we compare the result with the exact solution.

A visual impression of the exact magnetic field is given in the top panel of Fig. 1. For comparison the potential field used as initial condition for the iteration is shown in the middle panel of Fig. 1. One notices in particular that the potential field has a different connectivity than the analytical MHS solution. The numerical method will thus have to change the magnetic field connectivity during the iteration process.

In Fig. 2 we show $r-\theta$ cuts of pressure (left) and density (right) for the angle $\phi=0$. The top panels show these quantities for the exact solution, the middle panels for the initial condition and the bottom panels show the result of the numerical calculation. Visual inspection of these figures gives the impression that the method does achieve a good, but not perfect agreement with the exact solutions. Especially the regions close to the $\theta$ boundaries still show noticeable differences, which may be caused by the general problems with convergence closer to the poles due to the deformation of the numerical grid using spherical coordinates. A more sophisticated numerical grid, as discussed in Sect. 5, will probably help to overcome these problems.

To check the quality of the reconstruction in a more quantitative way we use a number of methods. The results are presented in Table 1. First, we evaluate how well the force balance
Table 1. The table provides several figures of merit which can be used to assess the quality of the reconstructed solution. We compute all figures for the complete computational domain. The analytical reference field was specified in the cones $11.25^{\circ} \leq \theta \leq 168.75^{\circ}$. For the calculation presented here we used the following iteration parameters: $\mu=1, v=$ $0.01, \xi=1$.

\begin{tabular}{c|lll}
\hline \hline & Ref. & Potential & Reconstruction \\
\hline$L$ & 0.003 & 0.004 & 0.002 \\
$L_{1}$ & 0.001 & 0.002 & 0.001 \\
$L_{2}$ & 0.002 & 0.002 & 0.001 \\
$\|\nabla \cdot \boldsymbol{B}\|_{\infty}$ & 0.448 & 0.582 & 0.448 \\
$\|\boldsymbol{j} \times \boldsymbol{B}-\nabla p-\rho \nabla \Psi\|_{\infty}$ & 0.137 & 0.186 & 0.143 \\
$C_{\mathrm{vec}}$ & 1 & 0.946 & 0.9997 \\
$C_{\mathrm{CS}}$ & 1 & 0.810 & 0.997 \\
$E_{N}$ & 0 & 0.378 & 0.021 \\
$E_{M}$ & 0 & 0.524 & 0.042 \\
$\epsilon$ & 1 & 0.951 & 1.0008 \\
Correlation $p$ & 1 & 0.975 & 0.9998 \\
Correlation $\rho$ & 1 & 0.928 & 0.9990 \\
No. of Steps & & & 214220 \\
Computing time & & & $12 \mathrm{~h} 27$ min \\
\hline
\end{tabular}

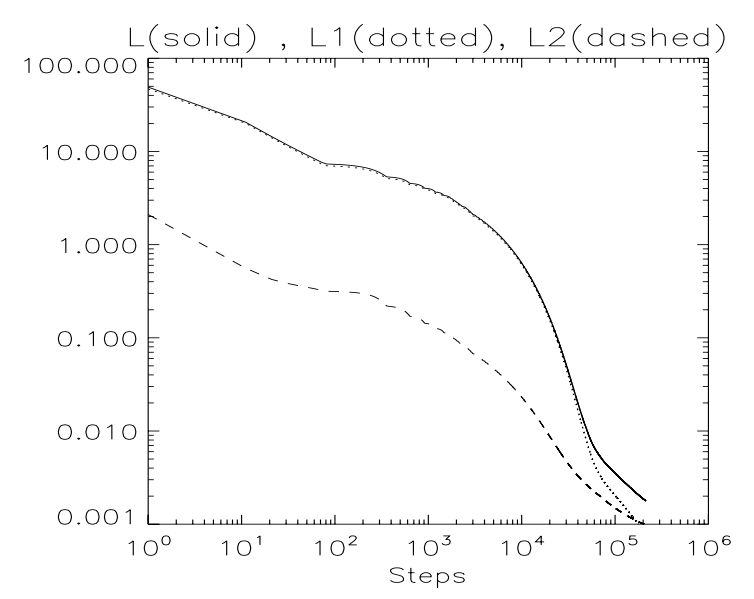

Fig. 3. Evolution of the functionals $L$ (solid line), $L_{1}$ (dotted line) and $L_{2}$ (dashed line; for definitions see text) during the iteration process. All three functionals decrease by several orders of magnitude during the iteration.

condition and the solenoidal condition are fulfilled. To do this we evaluate

- the functional $L$ as defined in (3),

- the functional $L_{1}=\int \frac{\left|(\nabla \times \boldsymbol{B}) \times \boldsymbol{B}-\mu_{0} \nabla p-\mu_{0} \nabla \Psi \rho\right|^{2}}{B^{2}} r^{2} \sin \theta \mathrm{d} r \mathrm{~d} \theta \mathrm{d} \phi$, telling us how well the force balance condition is satisfied,

- the functional $L_{2}=\int|\nabla \cdot \boldsymbol{B}|^{2} r^{2} \sin \theta \mathrm{d} r \mathrm{~d} \theta \mathrm{d} \phi$, which tells us how well the solenoidal condition is satisfied.

The evolution of the functionals $L, L_{1}, L_{2}$ during the numerical computation is shown in Fig. 3 in a double logarithmic plot. The functionals $L$ and $L_{1}$ decrease rapidly by about five orders of magnitude. The functional $L_{2}$, which represents the solenoidal condition decreases by about three orders of magnitude. The entries in the table give the values of the functionals at the end of the numerical calculation.

Following Amari et al. (2006) and Wiegelmann \& Neukirch (2006) we also provide the infinity norms

- $\|\nabla \cdot \boldsymbol{B}\|_{\infty}$

- $\|\boldsymbol{j} \times \boldsymbol{B}-\nabla p-\rho \nabla \Psi\|_{\infty}$, 


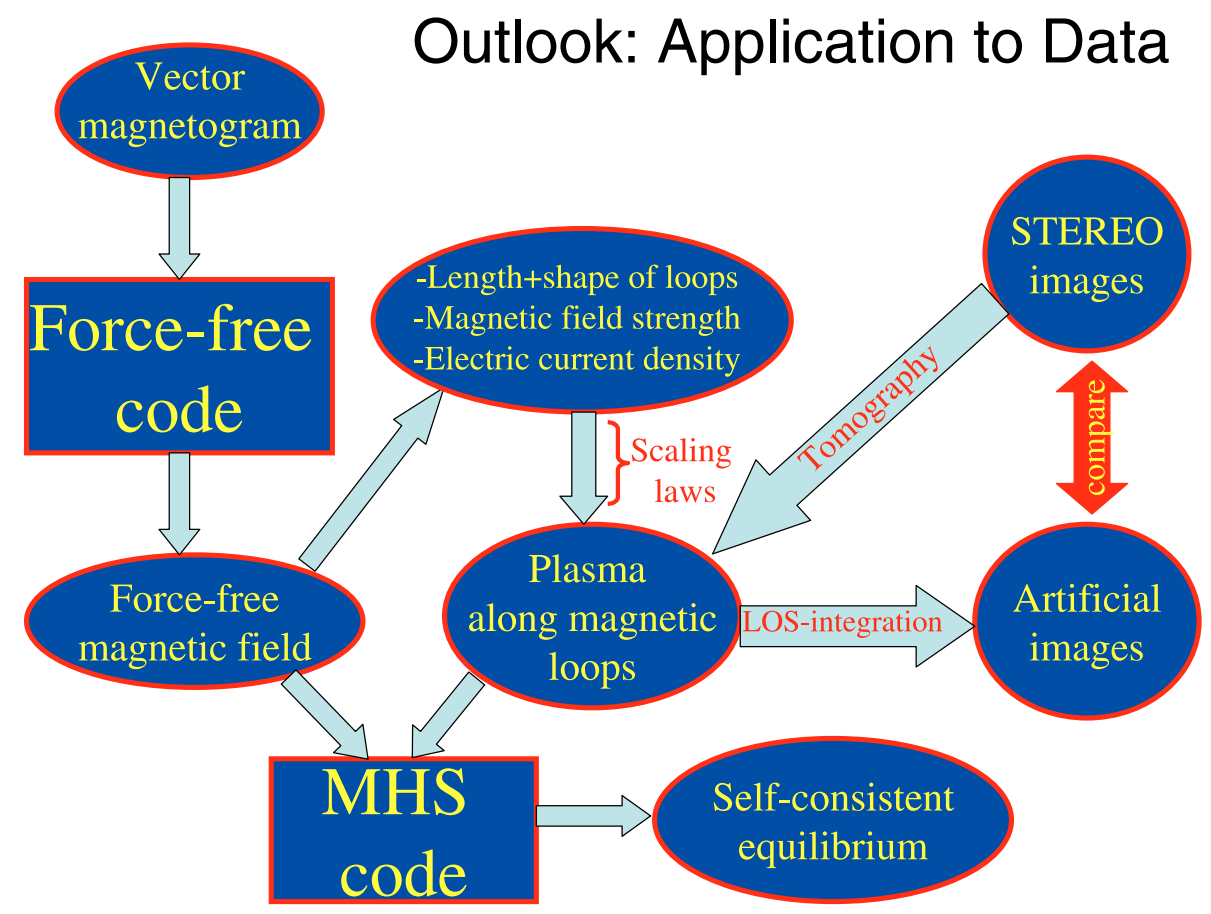

Fig. 4. Outlook: How can the tool become applied to data? The basic idea is to compute first a nonlinear forcefree field from observed vector magnetograms and then model the plasma along the loops. Our newly developed magnetohydrostatic code uses the resulting magnetic field and plasma configuration as input for the computation of a self-consistent equilibrium.

which are defined as the supremum of the divergence and the total force density. For comparison, we also provide these values for the exact analytic solution if used in a discretized form. This allows us to estimate the discretisation error introduced by calculating the solution on a finite numerical grid (Here 20, 40, 80 grid-points in the $r, \theta, \phi$ direction, respectively).

We compare the reconstructed equilibrium directly with the known analytic equilibrium by a number of quantitative measures as defined by Schrijver et al. (2006, see Sect. 4 of that paper). The figures quantify the difference between two discretized vector fields $\boldsymbol{B}$ (known analytic field evaluated on the numerical grid) and $\boldsymbol{b}$ (reconstructed field). These measures are:

- the vector correlation $C_{\mathrm{vec}}=\sum_{i} \boldsymbol{B}_{i} \cdot \boldsymbol{b}_{i} /\left(\sum_{i}\left|\boldsymbol{B}_{i}\right|^{2} \sum_{i}\left|\boldsymbol{b}_{i}\right|^{2}\right)^{1 / 2}$,

- the Cauchy-Schwarz inequality $C_{\mathrm{CS}}=\frac{1}{N} \sum_{i} \frac{\boldsymbol{B}_{i} \cdot \boldsymbol{b}_{i}}{\left|\boldsymbol{B}_{i}\right|\left|\boldsymbol{b}_{i}\right|}$, where $N$ is the number of vectors in the field;

- the normalized vector error $E_{\mathrm{N}}=\sum_{i}\left|\boldsymbol{b}_{i}-\boldsymbol{B}_{i}\right| / \sum_{i}\left|\boldsymbol{B}_{i}\right|$,

- the mean vector error $E_{\mathrm{M}}=\frac{1}{N} \sum_{i} \frac{\left|\boldsymbol{b}_{i}-\boldsymbol{B}_{i}\right|}{\left|\boldsymbol{B}_{i}\right|}$

- the magnetic energy of the reconstructed field divided by the energy of the analytical field $\epsilon=\frac{\sum_{i}\left|\boldsymbol{b}_{i}\right|^{2}}{\sum_{i}\left|\boldsymbol{B}_{i}\right|^{2}}$.

The quality of the reconstruction of the pressure $p$ and the density $\rho$ is quantitatively assessed by correlating the analytic and the reconstructed solutions using the linear Pearson correlation coefficients (called correlation $p$ and correlation $\rho$ in Table 1). Finally, we provide the number of iteration steps and computing time for a single processor run on a common workstation.

\section{Conclusions and outlook}

We have extended the optimization method originally proposed for the reconstruction of force-free magnetic fields (Wheatland et al. 2000) to global magnetohydrostatic equilibria including the pressure force and the gravitational force in spherical geometry. The proposed generalization of the optimization method leads to two additional equations for the pressure and the density that have to be solved simultaneously to the magnetic field equation.
Boundary conditions for the magnetic field, the pressure and the density are necessary to complete the problem.

We have implemented a numerical code based on the proposed method and have tested the code using a known threedimensional magnetohydrostatic equilibrium (Neukirch 1995). The numerical calculation is started from a potential field with the same radial magnetic field component as the analytical equilibrium on the boundary. The initial pressure and density distribution are a spherically symmetric stratified atmosphere in hydrostatic balance. Both visual inspection of the results as well as a quantitative analysis using various diagnostic measures indicate that the method works well and converges to the analytic equilibrium. For the presented tests we used a low spatial resolution and got a relatively long computing time (about 200000 iteration steps) until convergence. In experiments with the force-free version of our spherical code (see Wiegelmann 2007) we found that the computing time scales with $N^{5.4}$ regarding the number of grid points $N$ in one spatial direction. This is somewhat slower as the theoretical estimate of $N^{5}$ for a cartesian optimization code obtained by Wheatland et al. (2000). The spherical magnetohydrostatic code is significantly slower than the cartesian force-free code for two reasons.

1. The convergence of the numerical grid towards the poles requires a sufficiently small time-step.

2 . The plasma $\beta$ might vary strongly in the entire region and in particular low- $\beta$-regions require very small time-steps to compute the magnetic field and plasma simultaneously, because small changes in the Lorentz force can result in considerably large changes in the low $\beta$ plasma.

Point 1 . can be addressed by using a more sophisticated numerical grid, e.g. the Yin-Yang grid developed by Kageyama \& Sato (2004), which has been applied in geophysics (see e.g. Yoshida \& Kageyama 2004). This overset grid contains two complementary grids which lead to an almost uniformly spaced spherical grid. An additional advantage of the Yin-Yang grid is that it is suitable for massive parallelization. The Yin-Yang grid has been applied for geophysical simulations on the Earth simulator super-computer in Yokohama. To speed up the 2. point one 
might compute first the magnetic field alone as a nonlinear forcefree field (which is a reasonable approximation in low- $\beta$-regions) and switch on the self-consistent plasma iteration only after for a fine-tuning. A multi-scale approach, as recently implemented to speed up our force-free cartesian optimization code (see Metcalf et al. 2007, for details) is also an option worth trying for the spherical implementation of our force-free and magnetohydrostatic codes. We are confident, that the above mentioned potential for improvements together with a massive parallelization will allow us to apply our newly developed method to real data with a reasonable grid resolution.

In Fig. 4 we outline a scheme on how the code might be applied to data. As boundary conditions on pressure and density are not directly measured, we propose the following approach. The basic idea is to compute first a force-free magnetic field and then model the plasma along the magnetic loops, e.g., by the use of scaling laws and optionally with the help of a tomography code. Such an approach has been used by Schrijver et al. (2004) by using a global potential magnetic field and specifying free scaling law parameters (e.g., heating function) by comparing artificial plasma images (created by line-of-sight integration from the model plasma) with X-ray and EUV observations. We propose to generalize this approach by using a nonlinear force-free magnetic field model and compare the model plasma with observations from two viewpoints as provided by the STEREO-mission. Optional STEREO-images can be used additionally to approximate the coronal density distribution by a tomographic inversion. As a consequence of this step (modelling the plasma along a magnetic loop) the plasma pressure is consistent along the loops. Different values for the pressure on different field-lines will violate the force-free condition for the magnetic field, however, and the configuration is not exactly in a magnetohydrostatic equilibrium. Finite pressure gradients have to be compensated by a Lorentz force. This computation can be done with the help of the program described in this paper. We propose to use the force-free magnetic field configuration and the model plasma as initial state for our newly developed magnetohydrostatic code to compute a self-consistent MHS-equilibrium. For a low $\beta$ plasma the back-reaction of the plasma onto the magnetic field will be small, for higher values of $\beta$ (as found e.g., in helmet streamers) the magnetic field might change significantly. As a result of this approach one has reconstructed the 3D coronal magnetic field and plasma configuration self-consistently within the magnetohydrostatic approach and the model is consistent with measured photospheric vector magnetograms and observed coronal images from different viewpoints as well.

Acknowledgements. The work of T. Wiegelmann was supported by DLR-grant 50 OC 0501. T. Neukirch acknowledges financial support by STFC. The work of P. Ruan was supported by the International Max-Planck Research School on Physical Processes in the Solar System and Beyond at the Universities of Braunschweig and Goettingen. Financial support by the European Commission through the SOLAIRE Network (MTRN-CT-2006-035484) is also gratefully acknowledged. We would like to thank the referee, Mike Wheatland, for useful remarks to improve this paper.

\section{References}

Amari, T., Aly, J. J., Luciani, J. F., Boulmezaoud, T. Z., \& Mikic, Z. 1997, Sol. Phys., 174, 129

Amari, T., Boulmezaoud, T. Z., \& Aly, J. J. 2006, A\&A, 446, 691

Bogdan, T. J., \& Low, B. C. 1986, ApJ, 306, 271

Inhester, B., \& Wiegelmann, T. 2006, Sol. Phys., 235, 201

Kageyama, A., \& Sato, T. 2004, Geochemistry, Geophysics, Geosystems, 5, 9005

Low, B. C. 1982, ApJ, 263, 952

Low, B. C. 1985, ApJ, 293, 31

Low, B. C. 1991, ApJ, 370, 427

Low, B. C. 1992, ApJ, 399, 300

Low, B. C. 1993a, ApJ, 408, 689

Low, B. C. 1993b, ApJ, 408, 693

Low, B. C. 2005, ApJ, 625, 451

Metcalf, T. R., DeRosa, M. L., Schrijver, C. J., et al. 2007, Sol. Phys., submitted Neukirch, T. 1995, A\&A, 301, 628

Neukirch, T. 1997, A\&A, 325, 847

Neukirch, T., \& Rastätter, L. 1999, A\&A, 348, 1000

Petrie, G. J. D., \& Neukirch, T. 2000, A\&A, 356, 735

Schrijver, C. J., Derosa, M. L., Metcalf, T. R., et al. 2006, Sol. Phys., 235, 161

Schrijver, C. J., Sandman, A. W., Aschwanden, M. J., \& DeRosa, M. L. 2004, ApJ, 615, 512

Wheatland, M. S. 2006, Sol. Phys., 238, 29

Wheatland, M. S., Sturrock, P. A., \& Roumeliotis, G. 2000, ApJ, 540, 1150

Wiegelmann, T. 2004, Sol. Phys., 219, 87

Wiegelmann, T. 2007, Sol. Phys., 240, 227

Wiegelmann, T., \& Inhester, B. 2003, Sol. Phys., 214, 287

Wiegelmann, T., \& Neukirch, T. 2003, Nonlinear Processes in Geophysics, 10, 313

Wiegelmann, T., \& Neukirch, T. 2006, A\&A, 457, 1053

Yoshida, M., \& Kageyama, A. 2004, Geophys. Res. Lett., 31, 12609 